\title{
New assessment for advanced age: Italian study protocol on the assessment of surgical risk in the over-75-year age-group
}

\author{
Annarita Pilleri, Giampaolo Scorcu \\ Cardiovascular Department - Cardiac Evaluation Unit, G. Brotzu Hospital, Cagliari, Italy
}

\begin{abstract}
The role of age in the risk stratification of patients candidate for non-cardiac surgery is still today an unresolved issue. European guidelines, in contrast to American guidelines, do not attribute to age an independent role in increasing the risk, and the indices for assessment of perioperative cardiovascular risk are based on studies that were carried out on middle-aged subgroups of the population without specific attention to the elderly patient. While the indices of geriatric assessment have still not yet gained a standardized role in the risk stratification of patients candidate to non-cardiac surgery, their need is becoming increasingly urgent considering the epidemiological impact of elderly patients with multi-comorbidities who more and more in the future will undergo such interventions. The European guidelines themselves identify an "evidence gap" concerning frailty which requires a deeper evaluation. The aim of the multicenter observational study VALUTA-75 is to verify if the indices of risk stratification routinely used by the cardiologist integrated with those of physical and cognitive performance of specific geriatric pertinence can improve the ability to predict perioperative cardiovascular and non cardiovascular events, with the scope of improving the therapeutic process.
\end{abstract}

\section{Introduction}

Population ageing and the increased life expectancy have led in the last 10 years to an increase of the mean age of patients undergoing surgery, and the clinical impact of the elderly population can-

Corresponding author: Annarita Pilleri, Struttura Semplice Dipartimentale Consulenza e Valutation Cardiologica, Piazzale Alessandro Ricchi 1, Ospedale G. Brotzu, 09134 Cagliari, Italy. E-mail: annaritapilleri@aob.it

Key words: Perioperative risk; non-cardiac surgery; elderly population.

Received for publication: 30 November 2016

Accepted for publication: 7 December 2016

(C) Copyright A. Pilleri and G. Scorcu, 2017

Tipografia PI-ME Editrice, Italy

Monaldi Archives for Chest Disease 2017; 87:850

doi: 10.4081/monaldi.2017.850

This article is distributed under the terms of the Creative Commons Attribution Noncommercial License (by-nc 4.0) which permits any noncommercial use, distribution, and reproduction in any medium, provided the original author(s) and source are credited. didate for non-cardiac surgery will likely continue to increase in the near future. It is estimated that in Europe, by 2020, there will be a $25 \%$ increase in cases of non-cardiac surgery. In the same time period, the number of elderly patients will increase by $50 \%$ and they will have 4 times the need of surgery with respect to the rest of the population [1]. Hence, interventions on elderly patients with multicomorbidity [2] and with a high prevalence of cardiac diseases will increase progressively.

However, the role of age as a parameter in risk stratification is still not clear today: the ESC/ESA [3] guidelines do not attribute to age an independent role in increasing the risk of complications but they recognize age as related to the greater incidence of comorbidities and emergency interventions carried out in the elderly. On the contrary, the ACC/AHA [4] guidelines attribute to age a primary role as an independent predictor. In addition, the perioperative cardiovascular risk stratification scores currently recommended by the guidelines, the Revised Cardiac Risk Index of Lee (RCRI) [5] and the National Surgical Quality Improvement Program (NSQIP) [6], are based on studies conducted several years ago on middle-aged populations without specific attention to the elderly patient or to their clinical, functional and cognitive characteristics. This clinical scenario of lack of clear indications and strong epidemiological impact poses the problem of the need to identify with greater accuracy those elderly patients who can gain the most benefit from complex surgery; at the same time, there is a growing need to make better use of the scores currently in use, integrating them with new parameters to allow a better stratification of risk in all age-groups of the population undergoing surgery.

In this context, the role of assessment of the clinical syndrome of frailty - both physical and cognitive - is an emerging issue. Among the specific aspects of the frailty syndrome, of particular importance are the reduced functional capacity and the increased vulnerability to "stressors" [7] such as acute diseases or exacerbations of chronic diseases, or complex procedures such as a surgical intervention. A "stressor" such as a surgical operation exposes the frail elderly person to an increased risk of periprocedural complications, to a longer length of stay in hospital, and to functional decline towards disability and death [8]. The concept of functional capacity, which still today is the reference for identifying a reduced physiological reserve, was first introduced in the 2009 European guidelines on noncardiac surgery [9]. It is measured in metabolic equivalents (METs), and difficulty in climbing two ramps of stairs or running a short distance ( $<4$ METs) is associated to an increased incidence of postoperative cardiac events. Nevertheless, the high prevalence of comorbidities makes the subgroup of elderly patients extremely heterogeneous, and so functional capacity is often not determined or considered of little objective value and so not suitable for a complete assessment of functional status.

The geriatric indexes that measure physical and cognitive performance have still not yet gained a standardized place [10] in the assessment of the elderly patient with cardiovascular disease, and 
even less in the risk stratification of patients undergoing non-cardiac surgery. The European guidelines themselves identify an "evidence gap" regarding frailty which requires a more in-depth evaluation. The assessment of frailty through simple tests to classify the global functional status, neuropsychological status and physical performance is thus conceived as a process of good clinical practice [11], useful to characterize more accurately the risk of complications especially in complex clinical cases characterized by the presence of multi-comorbidities.

Based on these considerations and these uncertainties, the VALUTA-75 study has been designed to verify if the integration of routine risk scores with a multidimensional geriatric assessment can improve the prediction of short- and medium-term in-hospital outcomes (30 days, 6 months, 1 year) in a sample of elderly individuals candidate for non-cardiac surgery. In particular, the study will aim to verify if the measurement of global functional status with the Instrumental Activities of Daily Living (IADL) and Basic Activities of Daily Living (BADL) scales, cognitive status with the Mini-COG test, and physical performance with the Short Physical Performance Battery (SPPB) can give additive prognostic information concerning mortality and the onset of major complications, both cardiovascular complications (acute myocardial infarction, acute heart failure, ventricular fibrillation/cardiac arrest, $3^{\text {rd }}$ degree AV block, atrial fibrillation/flutter, angina, pulmonary embolism, ischemic/hemorrhagic stroke) and non cardiovascular complications (bleeding, transfusions, delirium, re-surgery, hospital length of stay, re-hospitalizations).

\section{Study design}

VALUTA-75 is a multicenter prospective observational study which will be carried out with the administrative authorization (verifying the feasibility) of each participating center and approval of the respective Ethics Committee. Approximately 3000 patients aged $\geq 75$ years undergoing elective non-cardiac surgery who are at moderate-high surgical risk according to ESC/ESA 2014 guidelines (Table 1) will be enrolled from 20 hospital centers equipped with adequate cardiac and geriatric facilities. Patients referred to the Cardiology Department for preoperative assessment will be informed about the aims and methods of the study and invited to participate. After signing (personally or by legal guardian) informed consent to participate in the study, patients will provide the name of a caregiver, for reference. Failure to provide written informed consent will be an exclusion criterion, as well as any physical or psychological disability that would render unfeasible the multidimensional assessment. Patients who are eligible will be enrolled consecutively, without any further selection criteria. Following routine cardiac assessments, patients will undergo a multidimensional evaluation (Table 2) by the geriatric équipe collaborating in the study. Data will be recorded on an electronic Case Report Form (eCRF) containing the patient's socio-demographic and clinical information and in which all data necessary for the study will be reported. Any significant event occurring during the patient's stay in hospital or any major perioperative complications will be recorded.

Table 1. Estimate of surgical risk (risk of myocardial infarction and cardiac death within 30 days post-surgery).

\begin{tabular}{lll} 
Low $<\mathbf{1} \%$ & Intermediate $\mathbf{1 - 5} \%$ & High $>5 \%$ \\
Plastic & Intraperitoneal & Major vascular \\
\hline Reconstructive & Symptomatic carotid artery & Peripheral vascular \\
\hline Breast & Peripheral angioplasty & Amputation \\
\hline Dental & Vascular endoprosthesis & Duodeno-pancreatic \\
\hline Endocrine: thyroid & Head and neck & Hepatectomy- biliary ducts \\
\hline Eye & Major orthopedic & Esophagectomy \\
\hline Asymptomatic carotid artery & Major urologic & Intestinal perforation \\
\hline Minor gynecological & Major gynecological & Total cystectomy \\
\hline Minor orthopedic & Renal transplant & Pneumonectomy \\
\hline Minor urologic & Minor intrathoracic & Liver/lung transplant
\end{tabular}

Table 2. Preoperative assessment.

\section{Cardiological}

1. Sex, age, weight, height and BMI

2. Type of surgery and its level of risk

3. Main diseases with special attention to cardiovascular comorbidities

4. Functional capacity measured in metabolic equivalents (METs)

5. Standard bio-humoral examinations: glycemia, urine albumin, total serum protein, hemoglobin, creatinine, glomerular filtration rate (GFR)

6. Estimate of cardiovascular risk through Revised Cardiac Risk Index of Lee (RCRI) and National Surgical Quality Improvement Program (NSQIP) for myocardial infarction and cardiac arrest (MICA)

7. Recommended treatment in relation to the risk estimation, risk factors and type of surgery

\section{Multidimensional geriatric}
1. Cumulative Illness Rating Scale (CIRS)
2. Instrumental Activities of Daily Living (IADL) and Basic Activities of Daily Living (BADL) scales
3. Mini-COG
4. Short Physical Perfomance Battery (SPPB) 
At 30 days post-surgery, there will be a first follow-up by phone to the patient or caregiver, in which the cardiologist/geriatrist will record the patient's vital status, enquire about eventual re-admissions and cardiovascular complications that have occurred, and evaluate the functional status through administration of IADL and BADL. The same procedure will be repeated at 6 and 12 months post-surgery (Table 3). Apart from the multidimensional geriatric assessment, no other pharmacological treatments or laboratory/instrumental examinations are planned aside from those normally used in each single center. The enrollment phase for each center will last 8 months starting from when the administrative authorization for the conduct of the study is granted. All enrolled patients with adequate data collection will be included in the analysis. Multivariate logistical analysis adjusting for age and the presence of baseline comorbidities will be performed to identify which markers are most predictive of outcome.

\section{Clinical considerations}

\section{Why plan a study such as VALUTA-75?}

The answer is complex, in line with the clinical complexity of the elderly patient. In dealing with elderly patients, clinical error and insufficient or inadequate treatment often stem from applying evidence from studies based on younger age-groups of the population or more simply from convictions based on experiences with, again, younger patients. And yet the demographic phenomenon represented by the general ageing of the population will increasingly place us in situations where we will have to face the complexity of elderly patients. This simple fact should prompt us to avoid errors such as limiting treatments or the scant conviction of their usefulness in elderly patients, but rather should prompt us to seek a better understanding of this biological frontier.

In line with their biological and demographic age, the prevalence of cardiovascular risk factors in the elderly (hypertension, diabetes, hypercholesterolemia) is very high, even though it is unknown in a large portion of the elderly population. Similarly, high is the prevalence of comorbidities such as ischemic heart disease, heart failure, cerebral small vessel disease, and frailty. The interaction of these variables (age, comorbidities and frailty) makes it difficult to formulate a precise diagnosis, and as a consequence therapy is often in- adequate or insufficient. Precisely this fact, however, imposes the need for accurate clinical research which is, above all, specific to this age-group.

The perioperative management of patients undergoing non-cardiac surgery is a problem that only recently has become the focus of scientific research. For instance, although the first ACC/AHA guidelines on the topic were published in 2002 [12], it was not until the update of 2007 [13] and the publication of the ESC/ESA guidelines in 2009 that there was any attempt to standardize the clinical approach through the use of an algorithm to trace a "road map" for patients undergoing surgery. Despite the publication of the new ESC/ESA guidelines in 2014, which define with precision the various types of intervention reclassifying their risk and modes of management, perioperative management still suffers from empirical, subjective and mechanistic attitudes. And if this is true in general, it is all the truer for elderly subgroups where the literature is still poor and the quantity of clinical studies available is truly miserable. Data are lacking on fundamental "clinical end points" in the elderly, such as heart failure and atrial fibrillation, and data are lacking on the impact of emerging non-cardiac risk factors such as frailty.

On account of population ageing, the study of frailty understood as a risk factor predisposing to cardiovascular complications cannot be neglected. The geriatric syndrome of frailty is, at the same time, a vast area with confines that are sometimes impossible to define: while it is easy to identify a disabled patient as frail, it is not always so easy to identify age-related conditions that are a source of vulnerability and thus an expression of frailty. Defining, then, the characteristics of frailty so that one can draw prognostic information from them must become a goal of daily clinical practice.

The field of research application of the VALUTA-75 study is to correlate the notion of frailty with exposure to a stressor such as a surgical intervention, with the scope of identifying new predictors of cardiovascular outcomes. VALUTA-75 is a multicenter observational study which hopes to provide an answer to some of the "evidence gaps" in the elderly population through a complex multidisciplinary collaboration involving cardiologists and geriatricians in the perspective of pursuit of a common goal.

If VALUTA-75 succeeds in producing the anticipated results, it will have made a step forward in improving the care of elderly patients and the criteria of selection of patients eligible for non-cardiac surgery. The information obtained will be useful for the prevention of cardiovascular and non cardiovascular events, and will improve the clinical appropriateness and use of the available healthcare resources.

Table 3. Postoperative assessments.

\section{In-hospital}

1. Length of hospital stay

2. Cardiovascular complications

3. In-hospital postoperative outcome

At 30 days (by phone contact or on occasion of the standard check-up visit)

1. Patient's vital status (alive/dead)

2. Cardiovascular complications or any re-admissions following discharge

At 6 and 12 months (by phone contact)

1. Patient's vital status (alive/dead)

2. Cardiovascular complications or re-admissions

3. Functional assessment through IADL and BADL scores 


\section{References}

1. Naughton C, Feneck R0. The impact of age on 6-month survival in patients with cardiovascular risk factors undergoing elective noncardiac surgery. Int J Clin Pract 2007;61:768-76.

2. Ferguson TB Jr, Hammill BG, et al. A decade of change-risk profiles and outcomes for isolated coronary artery bypass grafting procedures, 1990-1999: a report from the STS National Database Committee and the Duke Clinical Research Institute. Society of Thoracic Surgeons. Ann Thorac Surg 2002; 73:480-9; discussion 489-90.

3. Kristensen SD, Knuuti J, Saraste A, et al. 2014 ESC/ESA Guidelines on noncardiac surgery: cardiovascular assessment and management. The Joint Task Force on noncardiac surgery: cardiovascular assessment and management of the European Society of Cardiology (ESC) and the European Society of Anaesthesiology (ESA). Eur Heart J 2014; 35:2383-31.

4. Fleisher LA, Fleischmann KE, et al. ACC/AHA Guideline on Perioperative Cardiovascular Evaluation and Management of Patients Undergoing Noncardiac Surgery. J Am Coll Cardiol 2014; 64:e77-e137.

5. Lee TH, Marcantonio ER, Mangione CM et al. Derivation and prospective validation of a simple index for prediction of cardiac riskof major noncardiac surgery. Circulation 1999;100:1043-9.
6. Gupta PK, Gupta H, Sundaram A, et al. Development and validation of a risk calculator for prediction of cardiac risk after surgery. Circulation 2011;124:381-7.

7. Bergman H, Ferrucci L, Guralnik J, et al. Frailty: an emerging research and clinical paradigm dissues and controversies. J Gerontol A Biol Sci Med Sci 2007;62:731-7.

8. Shamliyan T, Talley KM, Ramakrishnan R, Kane RL. Association of frailty with survival: a systematic literature review. Ageing Res Rev 2012;12:719-36.

9. Poldermans D, Bax JJ, Boersma E, et al. Guidelines for preoperative cardiac risk assessment and perioperative cardiac management in noncardiac surgery. Eur Heart J 2009;30:2769-12.

10. Singh M, Stewart R, White H. Importance of frailty in patients with cardiovascular disease. Eur Heart J 2014;35:1726-31.

11. Afilalo J, Alexander KP, Mack MJ et al. Frailty assessment in the cardiovascular care of older adults. J Am Coll Cardiol 2014;63:747-62.

12. Eagle KA, Berger PB, Calkins, et al. ACC/AHA Guideline update for perioperative cardiovascular evaluation for noncardiac surgery. A report of the ACC/AHA Task Force on Practice Guidelines. J Am Coll Cardiol 2002;39:542-53.

13. Fleisher LA, Beckman JA, Brown KA, et al. ACC/AHA2007 Guidelines on perioperative cardiovascular evaluation and care for noncardiac surgery. A Report of the American College of Cardiology/American Heart Association Task Force on Practice Guidelines. J Am Coll Cardiol 2007;50:e159-241. 